\title{
The Effect of Temperature Dependent Viscosity on MHD Natural Convection Flow from an Isothermal Sphere
}

\author{
M.M. Molla ${ }^{1 \dagger}$, S.C. Saha ${ }^{2}$ and M.A. Hossain \\ ${ }^{I}$ Department of Electrical Engineering \& Computer Science, North South University, Dhaka1229, Bangladesh \\ ${ }^{2}$ School of Chemistry, Physics \& Mechanical Engineering, Queensland University of Technology, 2 George St., GPO \\ Box 2434, Brisbane QLD 4001, Australia \\ ${ }^{3}$ Department of Mathematic, COMSATS Institute of Information Technology, Islamabad, Pakistan
}

†Corresponding AuthorEmail:mmamun@northsouth.edu,mmamun@gmail.com

(Received February 28, 2010; Accepted July 19, 2010)

\begin{abstract}
Laminar magnetohydrodynamic (MHD) natural convection flow from an isothermal sphere immersed in a fluid with viscosity proportional to linear function of temperature has been studied. The governing boundary layer equations are transformed into a non-dimensional form and the resulting nonlinear system of partial differential equations are reduced to convenient form which are solved numerically by two very efficient methods, namely, (i) Implicit finite difference method together with Keller box scheme and (ii) Direct numerical scheme. Numerical results are presented by velocity and temperature distribution, streamlines and isotherms of the fluid as well as heat transfer characteristics, namely the local skin-friction coefficients and the local heat transfer rate for a wide range of magnetohydrodynamic paramagnet and viscosity-variation parameter.
\end{abstract}

Keywords: Natural convection, MHD, Temperature dependent viscosity, Finite difference, Sphere.

\section{NOMENCLATURE}

$\begin{array}{ll}a & \text { radius of the sphere } \\ C_{p} & \text { specific heat at constant pressure } \\ C_{f} & \text { skin-friction coefficient } \\ f & \text { dimensionless stream function } \\ g & \text { acceleration due to gravity } \\ G r & \text { Grashof number } \\ k & \text { thermal conductivity of the fluid } \\ M & \text { MHD parameter } \\ N u & \text { Nusselt Number } \\ \operatorname{Pr} & \text { Prandtl Number } \\ q_{w} & \text { heat flux at the surface } \\ T & \text { temperature of the fluid } \\ T_{\infty} & \text { temperature of the ambient fluid } \\ T_{w} & \text { temperature at the surface } \\ u, v & \text { dimensionless velocity components } \\ \hat{u}, \hat{v} & \text { dimensional velocity components }\end{array}$

\section{INTRODUCTION}

A study of the flow of electrically conducting fluid in presence of magnetic field is important from the technical point of view and such types of problems have received much attention by many researchers. Vajravelu and Hadjinolaou (1997) studied the convective heat transfer in an electrically conducting fluid at a stretching surface. As mentioned by Vajravelu and $\mathrm{x}, \mathrm{y}$ axis direction

$\beta \quad$ volumetric coefficient of thermal expansion

$\psi \quad$ stream function

$\tau_{\mathrm{w}} \quad$ shearing stress

$\gamma \quad$ viscosity-variation parameter

$\gamma^{*} \quad$ constant

$\rho \quad$ density of the fluid

$v \quad$ reference kinematic viscosity

$\mu(T)$ viscosity of the fluid

$\theta$ dimensionless temperature function

$\beta_{0} \quad$ strength of magnetic field

$\sigma_{0} \quad$ electric conduction

$\gamma^{*}$ constant
Hadjinolaou (1997), the rate of cooling and, therefore, the desired properties of the end product can be controlled by the use of electrically conducting fluids and the application of magnetic field. The use of magnetic field has been also used in the process of purification of molten metals from non-metallic inclusions. MHD free convection flow of visco-elastic fluid past an infinite porous plate was investigated by Chowdhury and Islam (2000). Raptis and Kafousian 
(1982) have investigated the problem of magnetohydrodynamic free convection flow and mass transfer through a porous medium bounded by an infinite vertical porous plate with constant heat flux.

\section{Formulation of Problem}

A steady two-dimensional MHD laminar free convective flow from a uniformly heated sphere of radius $a$, which is immersed in a viscous and incompressible fluid having temperature dependent viscosity. Here viscosity is the linear function of the fluid temperature. It is assumed that the surface temperature of the sphere $T_{w}>T_{\infty}$ where $T_{\infty}$ is the ambient temperature of the fluid. The configuration considered is as shown in Fig. 1.

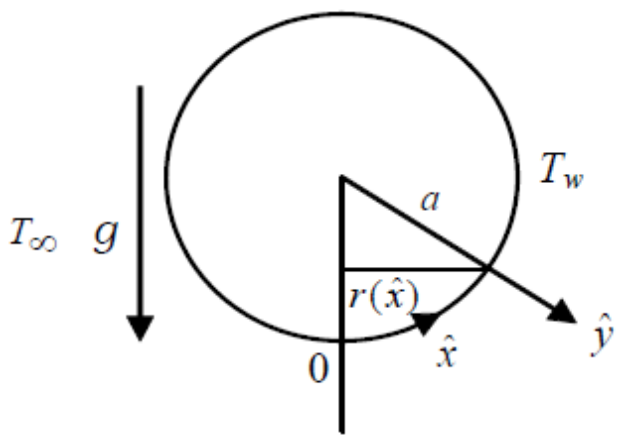

Fig. 1. Physical model and coordinate system.

The equations governing the flow are

$\frac{\partial}{\partial \hat{x}}(r \hat{u})+\frac{\partial}{\partial \hat{y}}(r \hat{v})=0$

$\hat{u} \frac{\partial \hat{u}}{\partial \hat{x}}+\hat{v} \frac{\partial \hat{u}}{\partial \hat{y}}=\frac{1}{\rho} \frac{\partial}{\partial \hat{y}}\left(\mu \frac{\partial \hat{u}}{\partial \hat{y}}\right)$

$$
+g \beta\left(T-T_{\infty}\right) \sin \left(\frac{\hat{x}}{a}\right)-\frac{\sigma_{0} \beta_{0}^{2}}{\rho} \hat{u}
$$

$\hat{u} \frac{\partial T}{\partial \hat{x}}+\hat{v} \frac{\partial T}{\partial \hat{y}}=\frac{k}{\rho C_{p}} \frac{\partial^{2} \hat{u}}{\partial \hat{y}^{2}}$

The boundary conditions of Eqs. (1) to (3) are

$\hat{u}=\hat{v}=0, \quad T=T_{w}$, at $\hat{y}=0$

$\hat{u} \rightarrow 0, \quad T \rightarrow T_{\infty}$ as $\hat{y} \rightarrow \infty$

where $r(\hat{x})=a \sin (\hat{x} / a),(\hat{u}, \hat{v})$ are velocity components along the $(\hat{x}, \hat{y})$ axes, $g$ is the acceleration due to gravity, $\rho$ is the density, $\mu(T)$ is the viscosity of the fluid depending on the fluid temperature $T, \beta$ is the coefficient of thermal expansion, $k$ is the thermal conductivity of the fluid, $\sigma_{0}$ is the electrical conduction, $\beta_{0}$ is the strength of magnetic field.

Out of the many forms of viscosity variation, which are available in the literature, we will consider only following form proposed by Charraudeau (1975)

$\mu=\mu_{\infty}\left[1+\gamma^{*}\left(T-T_{\infty}\right)\right]$ where $\mu_{\infty}$ is the viscosity of the ambient fluid and $\gamma^{*}$ is defined as follows

$\gamma^{*}=\frac{1}{\mu_{f}}\left(\frac{\partial \mu}{\partial T}\right)_{f}$

here $f$ denotes the film temperature of the fluid.

We now introduce the following non-dimensional variables:

$$
\begin{aligned}
& x=\frac{\hat{x}}{a}, y=G r^{1 / 4}\left(\frac{\hat{y}}{a}\right), u=\frac{\rho a}{\mu} G r^{-1 / 2} \hat{u} \\
& v=\frac{\rho a}{\mu} G r^{-1 / 2} \hat{v}, \quad \theta=\frac{T-T_{\infty}}{T_{w}-T_{\infty}}, \\
& G r=\frac{g \beta\left(T_{w}-T_{\infty}\right) a^{3}}{v_{\infty}^{2}}
\end{aligned}
$$

where $v_{\infty}\left(=\mu_{\infty} \rho\right)$ is the reference kinematic viscosity and $G r$ is the Grashof number and $\theta$ is the nondimensional temperature.

Substituting Eq. (6) into Eqs. (1)-(3) leads to the following non-dimensional equations

$$
\begin{aligned}
& \frac{\partial}{\partial x}(r u)+\frac{\partial}{\partial y}(r v)=0 \\
& \begin{aligned}
u \frac{\partial u}{\partial x}+v \frac{\partial u}{\partial y}= & (1+\gamma \theta) \frac{\partial^{2} u}{\partial y^{2}}+\gamma \frac{\partial u}{\partial y} \frac{\partial \theta}{\partial y} \\
& +\theta \sin x-M u
\end{aligned} \\
& \begin{aligned}
u \frac{\partial \theta}{\partial x}+v \frac{\partial \theta}{\partial y}= & \frac{1}{\operatorname{Pr}} \frac{\partial^{2} \theta}{\partial y^{2}}
\end{aligned}
\end{aligned}
$$

With the boundary conditions (4) become

$$
\begin{aligned}
& u=v=0, \quad \theta=1, \quad \text { at } \quad y=0 \\
& u \rightarrow 0, \quad \theta \rightarrow 0, \quad \text { as } y \rightarrow \infty
\end{aligned}
$$

where $\gamma$ is the viscosity-variation parameter, $M$ is the magnetic parameter and $\mathrm{Pr}$ is the Prandtl number which are defined as in Eq. (11), respectively

$$
\begin{aligned}
& \gamma=\frac{1}{\mu_{f}}\left(\frac{\partial \mu}{\partial T}\right)_{f}\left(T_{w}-T_{\infty}\right), \quad M=\frac{\sigma_{0} \beta_{0}^{2} a^{2}}{\mu_{\infty} G r^{1 / 2}} \\
& \text { and } \operatorname{Pr}=\frac{\mu c_{p}}{k}
\end{aligned}
$$

\section{Solution Methodology}

To solve the above non-linear differential equations we have employed two numerical methodologies: namely, implicit finite difference (IFD) method with the Keller box scheme and the direct numerical scheme (DNS), which are individually presented below.

\subsection{Implicit Finite Difference Method (IFD)}

Implicit finite difference (IFD) method was first introduced by Keller (1978) and elaborately described by Cebeci and Bradshaw (1984). To solve Eqs. (7)-(9) using IFD subject to the boundary conditions (10), we assume the following variables

$\psi=x r(x) f(x, y), \quad \theta=\theta(x, y)$ 
where $\psi$ is the non-dimensional stream function defined in the usual way as

$u=\frac{1}{r} \frac{\partial \psi}{\partial y}, \quad v=-\frac{1}{r} \frac{\partial \psi}{\partial x}$

Substituting Eqs. (12)-(13) into Eqs. (8)-(9) we get, after some algebra, the following transformed equations

$$
\begin{gathered}
(1+\gamma \theta) \frac{\partial^{3} f}{\partial y^{3}}+\left(1+\frac{x}{\sin x} \cos x\right) f \frac{\partial^{2} f}{\partial y^{2}} \\
+\gamma \frac{\partial^{2} f}{\partial y^{2}} \frac{\partial \theta}{\partial y}-\left(\frac{\partial f}{\partial y}\right)^{2}+\frac{\theta \sin x}{x}-M \frac{\partial f}{\partial y} \\
=x\left(\frac{\partial f}{\partial y} \frac{\partial^{2} f}{\partial x \partial y}-\frac{\partial f}{\partial x} \frac{\partial^{2} f}{\partial y^{2}}\right) \\
\frac{1}{\operatorname{Pr}} \frac{\partial^{2} \theta}{\partial y^{2}}+\left(1+\frac{x}{\sin x} \cos x\right) f \frac{\partial \theta}{\partial y} \\
=x\left(\frac{\partial f}{\partial y} \frac{\partial \theta}{\partial x}-\frac{\partial \theta}{\partial y} \frac{\partial f}{\partial x}\right)
\end{gathered}
$$

Along with boundary conditions

$$
\begin{aligned}
& f=\frac{\partial f}{\partial y}=0, \quad \theta=1, \quad \text { at } \quad y=0 \\
& \frac{\partial f}{\partial y} \rightarrow 0, \quad \theta \rightarrow 0, \quad \text { as } \quad y \rightarrow \infty
\end{aligned}
$$

The physical qualities of principle interest are shearing stress in terms of the skin-friction coefficient and the rate of heat transfer in terms of the Nusselt number, which can be written, in non-dimensional form as

$$
\begin{aligned}
& C_{f}=\frac{2 \tau_{w}}{\rho U_{\infty}^{2}}, \quad N u=\frac{a q_{w}}{k\left(T_{w}-T_{\infty}\right)} \\
& \text { where } \tau_{w}=\left(\mu \frac{\partial \hat{u}}{\partial \hat{y}}\right)_{\hat{y}=0}, \quad q_{w}=-k\left(\frac{\partial T}{\partial \hat{y}}\right)_{\hat{y}=0}
\end{aligned}
$$

Using Eqs. (6), (12) and (16), we get

$$
\begin{aligned}
& \frac{C_{f} G r^{1 / 4}}{2(1+\gamma)}=x \frac{\partial^{2} f(x, 0)}{\partial y^{2}} \\
& N u G r^{-1 / 4}=-\frac{\partial \theta(x, 0)}{\partial y}
\end{aligned}
$$

\subsection{Direct Numerical Scheme}

To incorporate the DNS method solving Eqs. (7)-(9) subject to the boundary conditions (10), we introduce the following new set of transformations.

$$
X=x, \quad Y=y, \quad U=\frac{u}{x}, \quad V=v
$$

Using Eq. (21) into Eqs. (7)-(10), we get

$$
\begin{aligned}
& X \frac{\partial U}{\partial X}+\left(1+X \frac{\cos X}{\sin X}\right) U+\frac{\partial V}{\partial Y}=0 \\
& X U \frac{\partial U}{\partial X}+V \frac{\partial U}{\partial Y}+U^{2} \\
& =(1+\gamma \theta) \frac{\partial^{2} U}{\partial Y^{2}}+\gamma \frac{\partial U}{\partial Y} \frac{\partial \theta}{\partial Y}+\theta \frac{\sin X}{X}-M U
\end{aligned}
$$

$X U \frac{\partial \theta}{\partial X}+V \frac{\partial \theta}{\partial Y}=\frac{1}{\operatorname{Pr}} \frac{\partial^{2} \theta}{\partial Y^{2}}$

The corresponding boundary conditions are

$$
\begin{aligned}
& U=V=0, \quad \theta=1 \text { at } X=0 \text { any } Y \\
& U=V=0, \quad \theta=1 \text { at } Y=0, \quad X>0 \\
& U \rightarrow 0, \quad \theta \rightarrow 0 \quad \text { as } Y \rightarrow \infty, \quad X>0
\end{aligned}
$$

Now Eqs. (22)-(24) subject to the boundary conditions (25) are discretised for direct numerical scheme (DNS) using central-difference for diffusion terms and the forward-difference for the convection terms, finally we get a system of tri-diagonal algebraic equations following as:

$A_{i} \phi_{i-1}+B_{i} \phi_{i}+C_{i} \phi_{i+1}=D_{i}$

where, $\phi$ is the dependent variable, $A_{i}, B_{i}, C_{i}$ are the tridiagonal matrix elements and $D_{i}$ are the nonhomogeneous terms of the associated matrix. The algebraic Eq. (26) have been solved by Gaussian elimination technique. In computation, the continuity equation has been solved directly for the normal velocity $V$ by the following discretisation:

$$
\begin{aligned}
& V_{i, j}=V_{i, j-1}-\Delta Y \frac{1}{2}\left(U_{i, j-1}+U_{i, j}\right)\left(1+X \frac{\cos X}{\sin X}\right) \\
& -X_{i} \frac{\Delta Y}{\Delta X}\left(U_{i, j}-U_{i-1, j}\right)
\end{aligned}
$$

The computation is started from $X=0.0$, and then marches up implicitly. Here $\Delta x=\pi / 180$ and $\Delta y=0.01$ are used for the $X$-and $Y$ - grids respectively.

Now we are at the position to measure of the physical quantities, namely the shearing stress and the rate of heat transfer from the following dimensionless relations:

$$
\begin{aligned}
& \frac{C_{f} G r^{1 / 4}}{2(1+\gamma)}=X\left(\frac{\partial U}{\partial Y}\right)_{Y=0} \\
& N u G r^{-1 / 4}=-\left(\frac{\partial \theta}{\partial Y}\right)_{Y=0}
\end{aligned}
$$

\section{RESUlt AND Discussion}

In this study we have investigated the problem of MHD laminar natural convection flow and heat transfer from an isothermal sphere with temperature dependent viscosity. Here we have considered the viscosity of the fluid is proportional to the linear function of temperature that means if the temperature of the fluid increases, the viscosity of the fluid also increases. This phenomenon generally occurs for small Prandtl number Pr. For example, the viscosity of air is $0.6924 \times 10^{-5}$ $\mathrm{kg} \cdot \mathrm{m}^{-1} \cdot \mathrm{s}^{-1}, 1.3289 \mathrm{~kg} \cdot \mathrm{m}^{-1} \cdot \mathrm{s}^{-1}, 2.286 \mathrm{~kg} \cdot \mathrm{m}^{-1} \cdot \mathrm{s}^{-1}$ and 3.625 $\mathrm{kg} \cdot \mathrm{m}^{-1} \cdot \mathrm{s}^{-1}$ at $\quad 100^{0} \mathrm{~K}, \quad 200^{0} \mathrm{~K}, \quad 400^{0} \mathrm{~K}$ and $800^{0} \mathrm{~K}$ temperature respectively. The viscosity of ammonia, $\mathrm{NH}_{3}$ is $7.255 \times 10^{-6} \mathrm{~kg} \cdot \mathrm{m}^{-1} \cdot \mathrm{s}^{-1}, 12.886 \mathrm{~kg} \cdot \mathrm{m}^{-1} \cdot \mathrm{s}^{-1}$, and $16.49 \mathrm{~kg} \cdot \mathrm{m}^{-1} \cdot \mathrm{s}^{-1}$ at $\quad 220^{0} \mathrm{~K}, \quad 373^{0} \mathrm{~K}$ and $473^{0} \mathrm{~K}$ respectively (see Cebeci and Bradshaw, 1984).

Equations (14)-(15) subject to the boundary conditions (16) are solved numerically using implicit finite- 
M.M. Molla et al. / JAFM, Vol. 5, No. 2, pp. 25-31, 2012.

Table 1 The results of $C_{f} G r^{1 / 4}$ for different values of the viscosity variation parameter $\gamma$ while $M=0.5$ and $\operatorname{Pr}=0.73$.

\begin{tabular}{ccccccccc}
\hline \multirow{2}{*}{$x^{0}$} & \multicolumn{9}{c}{$C_{f} G r^{1 / 4}$} \\
\cline { 2 - 8 } & \multicolumn{2}{c}{$\gamma=0.0$} & \multicolumn{2}{c}{$\gamma=1.0$} & \multicolumn{2}{c}{$\gamma=3.0$} & \multicolumn{2}{c}{$\gamma=5.0$} \\
\cline { 2 - 9 } & IFD & DNS & IFD & DNS & IFD & DNS & IFD & DNS \\
\hline 0.0 & 0.00000 & 0.00000 & 0.00000 & 0.00000 & 0.00000 & 0.00000 & 0.00000 & 0.00000 \\
30 & 0.34404 & 0.34476 & 0.21615 & 0.21665 & 0.13491 & 0.13524 & 0.10206 & 0.10232 \\
60 & 0.62442 & 0.62658 & 0.39306 & 0.39447 & 0.24578 & 0.24672 & 0.18612 & 0.18685 \\
90 & 0.78522 & 0.78929 & 0.49629 & 0.49895 & 0.31153 & 0.31326 & 0.23639 & 0.23774 \\
120 & 0.78033 & 0.78652 & 0.49734 & 0.50149 & 0.31470 & 0.31747 & 0.23983 & 0.24207 \\
150 & 0.56193 & 0.57009 & 0.36517 & 0.37063 & 0.23555 & 0.23924 & 0.18143 & 0.18448 \\
179 & 0.01463 & 0.03269 & 0.01034 & 0.02260 & 0.00730 & 0.01559 & 0.00603 & 0.01253 \\
\hline
\end{tabular}

Table 2 The results of $N u \mathrm{Gr}^{-l-14}$ for different values of the viscosity variation parameter $\gamma$ while $M=0.5$ and $\operatorname{Pr}=0.73$.

\begin{tabular}{|c|c|c|c|c|c|c|c|c|}
\hline \multirow{3}{*}{$x^{0}$} & \multicolumn{8}{|c|}{$N u G r^{-I-/ 4}$} \\
\hline & \multicolumn{2}{|c|}{$\gamma=0.0$} & \multicolumn{2}{|c|}{$\gamma=1.0$} & \multicolumn{2}{|c|}{$\gamma=3.0$} & \multicolumn{2}{|c|}{$\gamma=5.0$} \\
\hline & IFD & DNS & IFD & DNS & IFD & DNS & IFD & DNS \\
\hline 0.0 & 0.43075 & 0.43029 & 0.39456 & 0.39414 & 0.35605 & 0.35565 & 0.33307 & 0.33270 \\
\hline 30 & 0.42046 & 0.42059 & 0.38527 & 0.38552 & 0.34779 & 0.34789 & 0.32541 & 0.32549 \\
\hline 60 & 0.38972 & 0.39021 & 0.35752 & 0.35796 & 0.32313 & 0.32350 & 0.30256 & 0.30290 \\
\hline 90 & 0.33658 & 0.33738 & 0.30957 & 0.31033 & 0.28055 & 0.28127 & 0.26311 & 0.26383 \\
\hline 120 & 0.25699 & 0.25790 & 0.23775 & 0.23861 & 0.21676 & 0.21759 & 0.20401 & 0.20483 \\
\hline 150 & 0.14451 & 0.14574 & 0.13570 & 0.13686 & 0.12563 & 0.12677 & 0.11927 & 0.12045 \\
\hline 179 & 0.00151 & 0.00499 & 0.00157 & 0.00494 & 0.00162 & 0.00485 & 0.00167 & 0.00478 \\
\hline
\end{tabular}

difference (IFD) together with Keller box scheme and the Eqs. (22)-(25) are solved by DNS. The numerical solutions start at the lower stagnation point of the sphere, $x \approx 0$ and proceed round the sphere up to the upper stagnation point, $x \approx \pi$. Solutions are obtained for MHD parameter $M(=0.0,0.2,0.5,0.8,1.0)$ and for a wide range of values of the variable viscosity parameter $\gamma\left(=0.0,1.0,3.0,5.0\right.$.). Since the values of $f^{\prime \prime}(x, 0)$ or $(\partial U / \partial Y)_{Y=0}$ and $\theta^{\prime}(x, 0)$ or $(\partial \theta / \partial Y)_{Y=0}$ are known from the solutions of the coupled Eqs. (14)-(15) or (22)-(24), numerical values of the shearing stress in terms of skinfriction coefficient $C_{f} G r^{1 / 4} / 2(1+\gamma)$ from Eq. (19) or Eq. (28) and the heat transfer rate in terms of the Nusselt number $N u$ from Eq. (20) or Eq. (29) are calculated from lower stagnation point to upper stagnation point of the sphere.

Numerical values of $C_{f} G r^{1 / 4} / 2(1+\gamma)$ and $N u G r^{-1 / 4}$ are depicted in Tables 1-2 and Fig. 2. It should be noted that for constant viscosity we recover the problem that discussed by Huang and Chen (1987) considering $\operatorname{Pr}=$ 1.0 which is not shown here. For the writing simplicity, we have used the symbol $C_{f} G r^{1 / 4}$ instead of $C_{f} G r^{1 / 4} / 2(1+\gamma)$.

The effect of different values of magnetic parameter $M$ $(=0.0,0.2,0.5,0.8,1.0)$ on local skin friction coefficient $C_{f} G r^{1 / 4}$ and the local Nusselt number $\mathrm{NuGr}^{-}$ ${ }^{1 / 4}$ with the viscosity-variation parameter $\gamma=1.0$ and for $\operatorname{Pr}=0.73$ are illustrated in Fig 2. Here we notice that the agreement between the results obtained by using the Keller box method (IFD) and the direct numerical scheme (DNS) is excellent. It can easily be seen that with the effect of magnetic parameter $M$ leads to decrease the local skin friction coefficient $C_{f} G r^{1 / 4}$ and the local Nusselt number $N u G r^{-1 / 4}$. This phenomenon can easily be understood from the fact that the increasing values of magnetic parameter $M$, the Lorentz force, which oppose the flow, that means, decrease the velocity and temperature gradient and hence the local skin-friction coefficient $C_{f} G r^{1 / 4}$ and the local Nusselt number $\mathrm{NuGr} r^{-1 /}$.
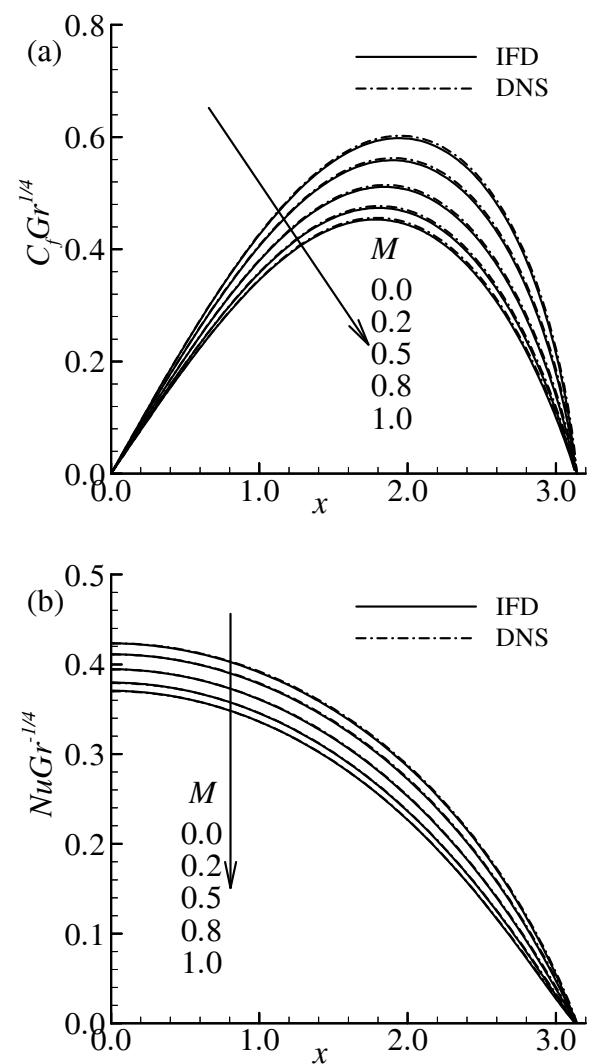

Fig. 2. (a) Skin-friction coefficient (b) Rate of heat transfer for different values of $\gamma$ while $\operatorname{Pr}=0.73$ and

$$
\varepsilon=1.0
$$

Owing to increase the values of increase the fluid temperature within the boundary layer and the associate 
thermal boundary layer becomes thicker. For increasing fluid temperature, the temperature difference between fluid and surface decreases and the corresponding rate of heat transfer $\mathrm{NuGr}^{-1 / 4}$ decreases. Here it should be noted that the point of separation of the flow occurs at the upper stagnation point $x \approx \pi$.

The numerical values of the skin-friction coefficient $C_{f} G r^{1 / 4}$ and the local Nusselt number $N u G r^{-1 / 4}$, against the curvature parameter $x$ for different values of viscosity-variation parameter $\gamma(=0.0,0.5,1.0,2.0,5.0)$ while $\mathrm{Pr}=0.73$ (air at $20^{\circ} \mathrm{C}$ and $1 \mathrm{~atm}$ pressure) are entered in Table 1 and 2 respectively. We further notice that, the agreement between the results obtained by the IFD method and the DNS is excellent. With the increasing values of the viscosity-variation parameter, it is seen that the values of skin-friction coefficient $C_{f} G r^{1 / 4}$ and the Nusselt number $N u G r^{-1 / 4}$ decrease. For increasing values of $\gamma$, the viscosity of the fluid within the boundary layer increases which retards the fluid motion, as a results the corresponding skin-friction coefficient $C_{f} G r^{1 / 4}$ decreases. For increasing values of the viscosity-variation parameter, the temperature of the fluid increase which is shown in Fig. 4b. Since the temperature of the fluid increases and hence the corresponding temperature difference between the surface and the fluid enhances. Due to higher temperature of the fluid the rate of heat transfer that means the Nusselt number $N u G r^{-1 / 4}$ decreases. It is important to calculate for the experimentalist that at $x=$ $\pi / 2$, the skin friction coefficient $C_{f} G r^{1 / 4}$ and the local Nusselt number $N u \mathrm{Gr}^{-1 / 4}$ decrease by $69.43 \%$ and $21.38 \%$ respectively as $\gamma$ increases from 0.0 to 5.0 .

Attention is now given to the effects of pertinent parameters on the dimensionless velocity and temperature in the flow field, computed only by the implicit finite difference (IFD) method, and these are presented graphically in Figs 3 and 4. Figure 3a-3b display results for the velocity and temperature profiles, based on Eqs. (14) and Eq. (15) with the boundary conditions (16), for different values of magnetic parameter $M(=0.0,0.2,0.5,0.8,1.0)$ plotted against $y$ at $x=\pi / 3$ having Prandtl number $\operatorname{Pr}=0.73$ with $\gamma=$ 1.0. From the Fig. 3 it is seen that, as the magnetic parameter $M$ increases, the velocity profile decrease and the temperature profile increases slightly. The reason of this practical scenario is that the interaction of the magnetic field and the moving electric charge carried by the fluid induces a force which tends to oppose the fluid motion. But near the surface of the cylinder, velocity increases and then decreases slowly and finally approaches to zero according to outer boundary condition. This implies that there exists a local maximum of the velocity within the boundary layer.

Figure $4 a-4 b$ illustrate the velocity and temperature distribution against the variable $y$ for different values of the viscosity-variation parameter $\gamma(=0.0,1.0,3.0,5.0)$ at $x=\pi / 3$ while $\operatorname{Pr}=0.73$ and $M=0.5$. It can be observed that the velocity decreases and temperature distribution increases with the increasing values of the viscosity-variation parameter, $\gamma$. It should be noted that at each value of the viscosity-variation-parameter $\gamma$, the velocity profile has a local maximum
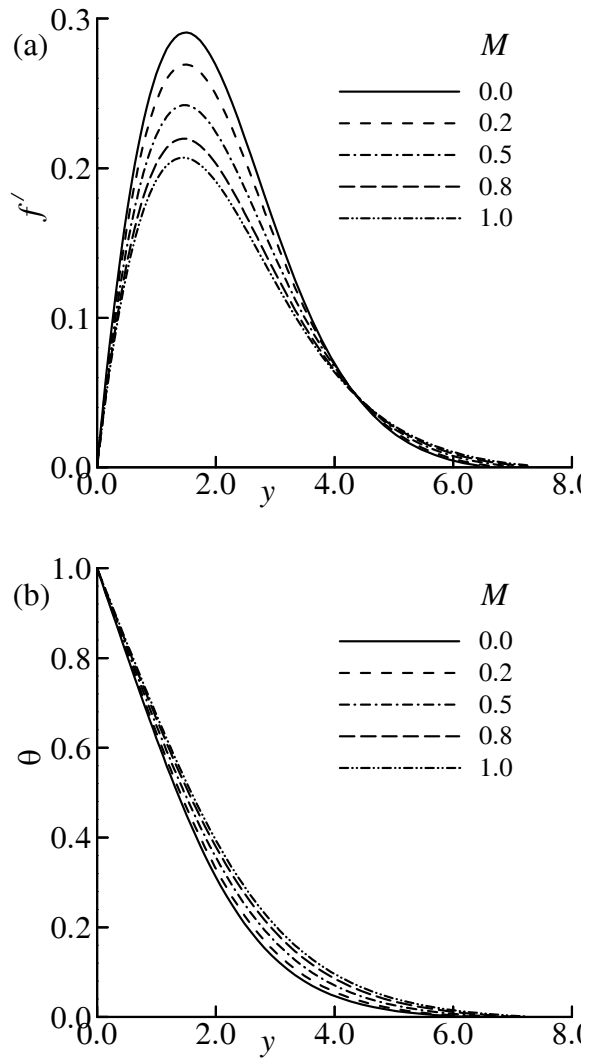

Fig. 3. (a) Velocity and (b) Temperature distribution for different values of $M$ while $\operatorname{Pr}=0.73$ at $x=\pi / 3$.
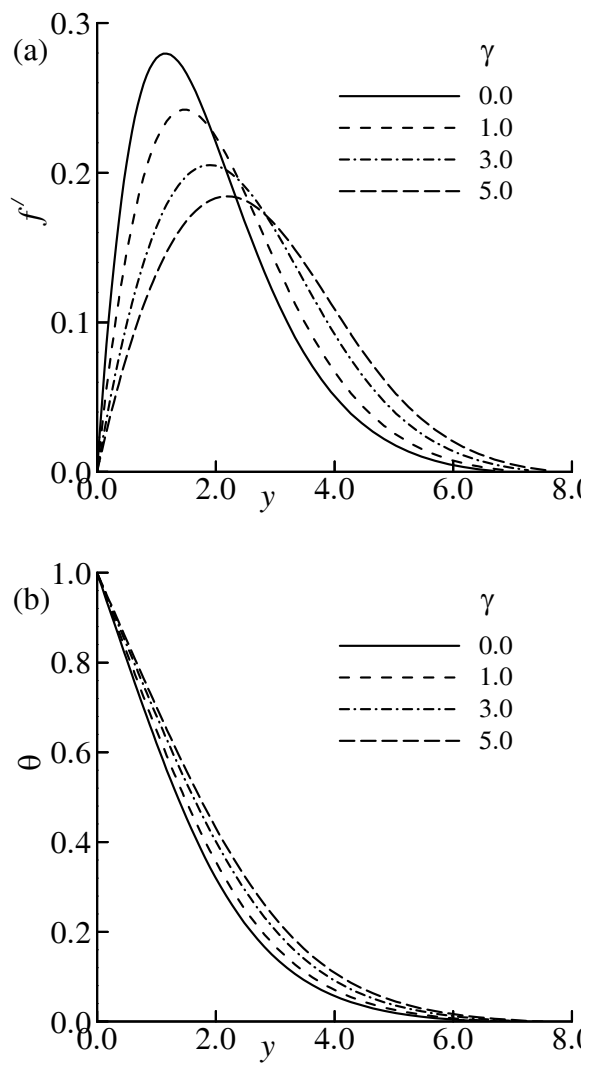

Fig. 4. (a) Velocity and (b) Temperature distribution for different values of $\gamma$ while $\operatorname{Pr}=0.73$ at $x=\pi / 3$. 
value within the boundary layer. The maximum values of the velocity are $0.33777,0.29494,0.25200,0.22745$ at $y=1.30254,1.65930,2.17434,2.48059$ for $\gamma=0.0$, $1.0,3.0,5.0$ respectively. The maximum velocity decreases by $32.66 \%$ as $\gamma$ increases from 0.0 to 5.0. It also be concluded that the velocity boundary layer and the thermal boundary layer thickness enhance for large values of $\gamma$.
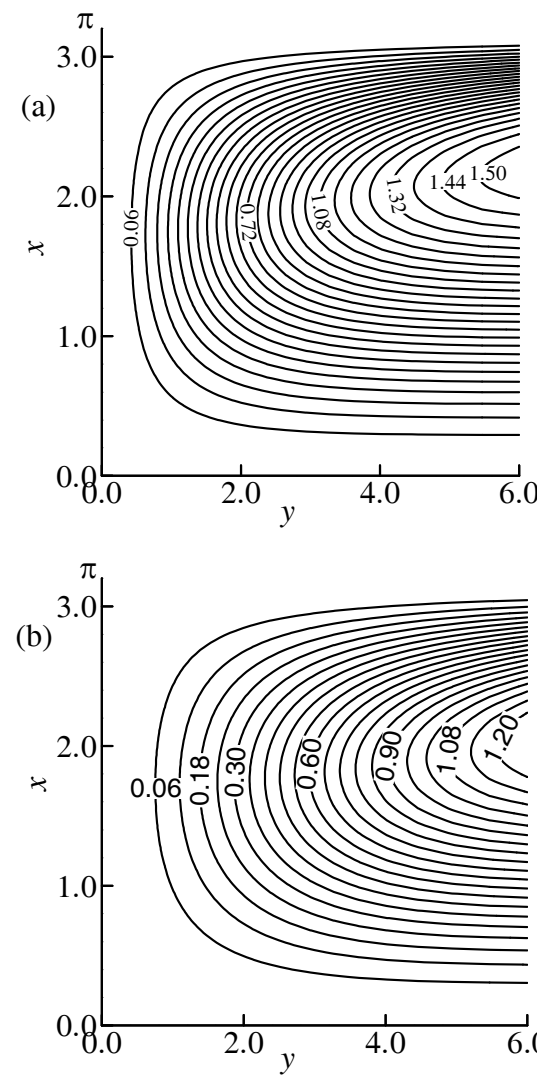

Fig. 5. Streamlines for (a) $\gamma=0.0$ and (b) $\gamma=5.0$ while $\operatorname{Pr}=0.73, M=0.5$ and $\Delta \psi=0.06$.

Figures 5 and 6 illustrate the effect of the viscosityvariation parameter, $\gamma$ on the development of streamlines and isotherms, which are plotted for $\operatorname{Pr}=$ 0.73 and $M=0.5$. From Fig. 5a, it is seen that without effect of viscosity-variation (i.e. $\gamma=0.0$ ) the nondimensional value of $\psi_{\max }$ within the computational domain is about 1.50 near at the upper stagnation point $(x \approx \pi)$ of the sphere and when the boundary layer thickness is the highest, but $\psi_{\max }$ decreases with the increment of $\gamma$ and it attains about 1.20 for $\gamma=5.0$ (see Fig. 5b). This phenomenon fully coincides with the early discussion made on Fig. 4a, the fluid slow down as $\gamma$ increases and the thickness of the velocity boundary layer also decreases.

The isotherm patterns for corresponding values of $\gamma$ are shown in Fig. 6. From these two frames, we can see that the growth of thermal boundary layer over the surface of the sphere in significant. As $x$ increases from the lower stagnation point $(x \approx 0.0)$, the hot fluid raises due to the gravity hence the thickness of the thermal boundary layer, $y$, increases. This phenomenon is very straightforward as can be seen in this frame for $\gamma=5.0$ in Fig. $5 \mathrm{~b}$ and in this case the fluid temperature increases slightly which was also noticed in Fig. $3 \mathrm{~b}$ and the surface heat transfer rate reduced (see in Table 2).
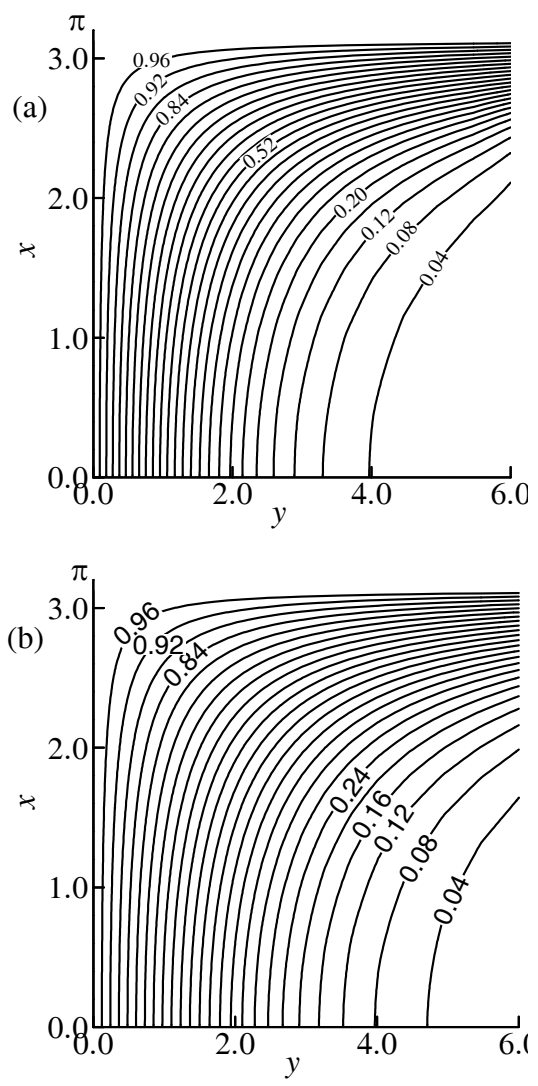

Fig. 6. Isotherms for (a) $\gamma=0.0$ and (b) $\gamma=5.0$ while $\operatorname{Pr}=0.73, M=0.5$ and $\Delta \theta=0.04$.

\section{CONCLUSION}

The effect of temperature-dependent viscosity on the MHD natural convection boundary layer flow from an isothermal sphere has been investigated theoretically. Numerical solutions of the equations governing the flow are obtained by using the very efficient implicit finite difference (IFD) method together with Keller box scheme and by a direct numerical scheme (DNS). From the present investigation the following conclusions may be drawn:

- Increasing the values of the magnetic parameter $M$ and viscosity-variation parameter $\gamma$ lead to decrease the local skin-friction coefficient $C_{f} G r^{1 / 4}$ and the local Nusselt number, $\mathrm{NuGr} r^{-1 / 4}$.

- It is seen the velocity distribution decrease as well as the temperature distribution increases with the increasing values of the magnetic parameter $M$ and viscosity-variation parameter.

- The results have demonstrated that the assumption of constant fluid properties may introduce severe errors in the prediction of the surface shearing stress and the rate of heat transfer. 


\section{REFERENCES}

Cebeci, T., and P. Bradshaw (1984). Physical and Computational Aspects of Convective heat Transfer. Springer, New York.

Charraudeau, J. (1975). Influence de gradients de properties physiques en convection force application au cas du tube. Int. J. Heat Mass Trans. 18, 87-95.

Chen, T.S., and A. Mocoglu (1977). Analysis of mixed forced and free convection about a sphere. Int. J. Heat Mass Transfer 20, 867-875.

Cheng C.Y. (2010). Natural convection boundary layer flow of fluid with temperature-dependent viscosity from a horizontal elliptical cylinder with constant surface heat flux. Applied Mathematics and Computation, In Press.

Cheng, C.Y. (2009). Natural convection heat transfer from a horizontal isothermal elliptical cylinder with internal heat generation. International Communications in Heat and Mass Transfer 36, 346-350.

Chiang, T., A. Ossin., and C.L. Tien (1964). Laminar free convection from a sphere. ASME J. Heat Transfer 86, 537-542.

Chowdhury, M.K., and M.N. Islam (2000). MHD free convection flow of visco-elastic fluid past an infinite porous plate. Heat and Mass Transfer 36, 439-447.

Hossain, M.A., M.S. Munir, and D.A.S. Rees (2000). Flow of viscous incompressible fluid with temperature dependent viscosity and thermal conductivity past a permeable wedge with uniform surface heat flux. Int. J. Therm. Sci. 39, 635-644.

Hossain, M.A., K.C.A Alam, and D.A.S. Rees (1997). MHD forced and free convection boundary layer flow along a vertical porous plate. Applied Mechanics and Engineering 2(1), 33-51.

Hossain, M.A., and M. Ahmed (1990). MHD forced and free convection boundary layer flow near the leading edge. Int. J. Heat Mass Transfer 33(3), 571-575.

Hossain, M.A., M.S. Munir, and R.S. Gorla (2002). Combined convection from a vertical flat plate with temperature dependent viscosity and thermal conductivity. Int. J. Fluid Mech. Res. 29(6), 725741 .

Huang, M.J., and C.K. Chen (1987). Laminar free convection from a sphere with blowing and suction. ASME J. Heat Transfer 109, 529-532.

Keller, H.B. (1978). Numerical methods in boundary layer theory, Annual Rev. Fluid Mech. 10, 417433.
Molla, M.M., M.A. Hossain and R.S.R. Gorla (2009). Natural Convection Laminar Flow with Temperature Dependent Viscosity and Thermal Conductivity along a Vertical Wavy Surface. International Journal of Fluid Mechanics Research 36, 272-288.

Molla, M.M, M.A. Hossain, and M.A. Taher (2006). Magnetohydrodynamic natural convection flow on a sphere with uniform heat flux in presence of heat generation. Acta Mechanica 186, 75-86.

Molla, M.M, M.A. Hossain, and R.S.R. Gorla (2005). Natural convection flow from an isothermal horizontal circular cylinder with temperature dependent viscosity. Heat Mass Transfer 41, 594598.

Molla, M.M., M.A. Taher, M.K. Chowdhury, and M.A. Hossain (2005). Magnetohydrodynamic natural convection flow on a sphere in presence of heat generation. Nonlinear Analysis: Modelling and Control 10(4), 349-363.

Molla, M.M, M.A. Hossain, and M.R.S. Gorla (2004). Conjugate effect of heat and mass transfer in natural convection flow from an isothermal sphere with chemical reaction. Int. J. Fluid Mech. Res. 31(4), 319-331.

Mukhopadhyay, S. (2009). Effects of radiation and variable fluid viscosity on flow and heat transfer along a symmetric wedge. Journal of Applied Fluid Mechanics, 2, 29-34.

Nazar, N., T. Grosan, N. Amin, Pop I. (2002). Free convection boundary layer on an isothermal sphere in a micropolar fluid, Int. Comm. Heat Mass Transfer 29(3), 377-386.

Raptis, A., and N. Kafousian (1982). Magnetohydrodynamic free convection flow and mass transfer through a porous medium bounded by an infinite vertical porous plate with constant heat flux. Canadian Journal of Physics 60(12), 1725-1729.

Sharma, P.R., and G. Singh (2009). Effects of variable thermal conductivity and heat source / sink on MHD flow near a stagnation point on a linearly stretching sheet. Journal of Applied Fluid Mechanics 2(1), 13-21.

Uddin, Z., and M. Kumar (2009). Effect of temperature dependent properties on MHD free convection flow and heat transfer near the lower stagnation point of a porous isothermal cylinder. Computer Modelling and New Technologies 13, 15-20.

Vajravelu, K., and A. Hadjinicolaou (1997). Convective heat transfer in an electrically conducting fluid at a stretching surface with uniform free stream. Int. J. Eng. Sci. 35, 1237-1244. 